\title{
Cluster observations of surface waves on the dawn flank magnetopause
}

\author{
C. J. Owen ${ }^{1}$, M. G. G. T. Taylor ${ }^{1,4}$, I. C. Krauklis ${ }^{1}$, A. N. Fazakerley ${ }^{1}$, M. W. Dunlop ${ }^{2,5}$, and J. M. Bosqued ${ }^{3}$ \\ ${ }^{1}$ Mullard Space Science Laboratory, Department of Space and Climate Physics, University College London, Holmbury St. \\ Mary, Dorking, Surrey, RH5 6NT, UK \\ ${ }^{2}$ Blackett Laboratory, Imperial College of Science Technology and Medicine, Prince Consort Road, London, SW7 2BZ, UK \\ ${ }^{3}$ CESR/CNRS, BP 4346 9, Avenue Colonel Roche, 31028 Toulouse Cedex, France \\ ${ }^{4}$ Now at Los Alamos National Laboratory, Los Alamos, NM, USA \\ ${ }^{5}$ Now at Rutherford Appleton Laboratory, Didcot, Oxon, UK
}

Received: 5 November 2002 - Revised: 2 June 2003 - Accepted: 24 July 2003 - Published: 19 March 2004

\begin{abstract}
On 14 June 2001 the four Cluster spacecraft recorded multiple encounters of the dawn-side flank magnetopause. The characteristics of the observed electron populations varied between a cold, dense magnetosheath population and warmer, more rarified boundary layer population on a quasi-periodic basis. The demarcation between these two populations can be readily identified by gradients in the scalar temperature of the electrons. An analysis of the differences in the observed timings of the boundary at each spacecraft indicates that these magnetopause crossings are consistent with a surface wave moving across the flank magnetopause. When compared to the orientation of the magnetopause expected from models, we find that the leading edges of these waves are approximately $45^{\circ}$ steeper than the trailing edges, consistent with the Kelvin-Helmholtz (KH) driving mechanism. A stability analysis of this interval suggests that the magnetopause is marginally stable to this mechanism during this event. Periods in which the analysis predicts that the magnetopause is unstable correspond to observations of greater wave steepening. Analysis of the pulses suggests that the waves have an average wavelength of approximately $3.4 R_{E}$ and move at an average speed of $\sim 65 \mathrm{~km} \mathrm{~s}^{-1}$ in an anti-sunward and northward direction, despite the spacecraft location somewhat south of the GSE $Z=0$ plane. This wave propagation direction lies close to perpendicular to the average magnetic field direction in the external magnetosheath, suggesting that these waves may preferentially propagate in the direction that requires no bending of these external field lines.
\end{abstract}

Key words. Magnetospheric physics (magnetospheric configuration and dynamics; MHD waves and unstabilities; solar wind-magnetosphere interactions)

Correspondence to: C. J. Owen

(cjo@mssl.ucl.ac.uk)

\section{Introduction}

Variations in the upstream solar wind velocity, pressure and in the interplanetary magnetic field act on the magnetopause boundary, such that the latter may be in almost continuous motion. The extent of this motion ranges from largescale surface motions to small waves or ripples (Kivelson and Chen, 1995; Seon et al., 1995; Sibeck et al., 1999). It is now widely accepted that magnetic reconnection or tearing (Dungey, 1961; Russell and Elphic, 1979) is the most efficient means of transferring solar wind plasma across the magnetopause (Sibeck et al., 1999). However, for low-shear magnetic field configurations, where the fields on either side of the magnetopause are more nearly parallel, reconnection is less likely to occur at low-latitudes, and other mechanisms, such as diffusion (Truemann et al., 1995) and "viscous" mechanisms (Axford and Hines, 1961; Miura, 1984) may become relatively more important.

First suggested by Dungey (1955), the Kelvin-Helmholtz instability (KHI) (Drazin and Reid, 1985) is a prime candidate for such an alternative energy transfer mechanism (Otto and Fairfield, 2000; Fairfield et al., 2000; Nykyri and Otto, 2001). The KHI, or "wind-over-water" instability may occur when two fluids are in motion with respect to one another. In this case, the stability of the interface between the two fluids to growth of boundary waves is dependent on the magnitude of the velocity shear. Such a mechanism may occur at the flank magnetopause boundary, where the fast-flowing plasma in the external magnetosheath slides past more stagnant magnetospheric or boundary layer populations, creating the velocity shear across the magnetopause necessary to invoke the instability. The growth of waves from the instability is dependent on the local fluid conditions. In particular, in a classical fluid, surface tension effects tend to counter the wave growth. In the case of the magnetized plasmas in and 
around the magnetosphere (Southwood, 1968), the surface tension effects are mimicked by the action of the magnetic field, which also controls the development of the KHI (Chandrasekhar, 1961).

A significant volume of work exists on the role of the KHI at the magnetopause, from a theoretical (Dungey, 1955; Southwood, 1968; Southwood and Hughes, 1983; Pu and Kivelson, 1983a, b), observational (Ogilvie and Fitzenreiter, 1989; Fitzenreiter and Ogilvie, 1995; Kivelson and Chen, 1995; Fairfield et al., 2000) and simulation point of view (Muira, 1995 and references therein, Otto and Fairfield, 2000; Nykyri and Otto, 2001). A comprehensive case study of magnetopause waves was carried out by Chen et al. (1993) (see also Chen and Kivelson, 1993; Kivelson and Chen, 1995), where they compared boundary observations made by ISEE- 1 and -2 to MHD simulations reported by Miura (1990). These authors determined the normals of the inbound and outbound magnetopause crossings by calculating the cross product of vectors measured simultaneously on opposite sides of the boundary. They suggested that the results were consistent with a symmetric tilting of the normals, such that the boundary wave would be of a non-sinusoidal nature. Interestingly, the results were consistent with the leading, downtail edges of the boundary waves being much shallower (i.e. less inclined from a nominal magnetopause orientation) than the trailing, sunward-facing edge. The waveform is thus somewhat akin to a wedge shape moving tailwards, with the thinnest edge leading. These observational results are at odds with theory, which suggests that the steepenedface of the boundary wave should be at the leading (more tailward) edge (Miura, 1990). However, more recently, Fairfield et al. (2000) compared GEOTAIL observations to the MHD simulation results from a companion paper by Otto and Fairfield (2000). In this case, the observations were consistent with the simulations, with the steepening of the leading edge of the waves (downtail side), as predicted by KHI theory. The reason for this discrepancy is not clear. Kivelson and Chen (1995) suggest that the magnetic curvature forces at the low shear boundary induce these steepened trailing edges. Fairfield et al. (2000) raise a question about the applied methods of determining the boundary normal directions in Chen et al. (1993); small-scale variations along the boundary and the incorrect determination of the boundary dimensions itself (compared to the separation scale of the spacecraft) could produce the erroneous results in the two-spacecraft method applied by Chen et al. (1993).

The simplest description of boundary wave growth under the action of the KHI is obtained by linearizing the ideal MHD momentum and induction equations (e.g. Southwood and Hughes, 1983; Treumann and Baumjohann, 1997), assuming that the length scale of the velocity shear (i.e. the boundary thickness) is much smaller than the wavelength of the driven boundary waves. Here, we consider the simple case of an incompressible plasma in which the group and phase velocity of the driven boundary waves are parallel to the magnetopause (e.g. Pu and Kivelson, 1983a). For this case, the condition for onset of the KHI and wave growth is given by:

$$
\left[\boldsymbol{k} \cdot\left(\boldsymbol{V}_{\mathbf{1}}-\boldsymbol{V}_{\mathbf{2}}\right)\right]^{2}>\frac{n_{1}+n_{2}}{\mu_{0} m_{p} n_{1} n_{2}}\left[\left(\boldsymbol{k} \cdot \boldsymbol{B}_{\mathbf{1}}\right)^{2}+\left(\boldsymbol{k} \cdot \boldsymbol{B}_{\mathbf{2}}\right)^{2}\right]
$$

(e.g. Chandrasekhar, 1961), where $\boldsymbol{V}$ is the plasma flow velocity, $n$ the plasma number density, $\boldsymbol{B}$ the magnetic field vector, $m_{p}$ is the proton mass, $\boldsymbol{k}$ is the wave vector and the subscripts refer to the two regions on either side of the boundary. For a flow shear $\left|\boldsymbol{V}_{\mathbf{1}}-\boldsymbol{V}_{\mathbf{2}}\right|<(>) V_{\mathrm{CRIT}}$, a critical value, we have stability (instability) and hence, no wave growth (wave growth). Note that $V_{\text {CRIT }}$ is dependent on both the magnetic field strength and orientation. In a configuration in which the magnetic fields on either side are perpendicular to the flow, a deformation of the shear layer boundary perturbs the magnetic field but in such a way that there is no tension force, and $V_{\text {CRIT }}$ is effectively identical to that expected in the case of an unmagnetized fluid. However, for a flow and field parallel to one another, the bending of the magnetic field during the growth of the waves will be opposed by the magnetic tension of the field. This damps the wave growth and thus effectively increases the magnitude of the critical velocity, $V_{\text {CRIT }}$.

The inclusion of considerations of plasma compressibility in the above analysis (e.g. Southwood, 1968; Pu and Kivelson, 1983a, b) adds complication to the behaviour of the system. In particular, more than a single mode of surface wave may arise, resulting in propagation perpendicular to the boundary, and upper and lower bounds on the critical velocity, depending on the sonic and magnetosonic speeds (e.g. Pu and Kivelson, 1983a). Southwood (1968) showed that the introduction of compressibility reduces the critical velocity relative to that in the incompressible case. However, in the case of super-Alfvénic flows, the compressibility has a stabilizing effect. It should also be noted that some work on this topic has considered the effects of plasma variations in the direction normal to the magnetopause, or where the scale thickness of the plasma transition may be of the order of, or larger than, the wavelength of the boundary waves. In particular, a number of authors (Ogilvie and Fitzenreiter, 1989; Kivelson and Chen, 1995; Farrugia et al., 1998, 2000) have pointed out that the magnetopause itself may not be Kelvin-Helmholtz unstable, while the inner edge of the magnetopause boundary layer, located somewhat deeper inside the magnetosphere, may satisfy the instability criterion. However, in the present study we concentrate only on observations of boundary waves at the magnetopause itself and so consider only the incompressible case.

In this paper, we present Cluster observations of surface waves on the dawn flank magnetopause. We utilize the novel and unique four-point measuring capability of this mission to examine the 3-D morphology of these features on the scale of the inter-spacecraft separations. The principal mission of Cluster is to resolve spatial-temporal ambiguities, such as those discussed above (e.g. Fairfield et al., 2000), that have arisen from studies of single (and double) spacecraft observations (e.g. the Chen et al., 1993 "reversed" wave profile). In particular, we compare the observed times of the transitions 


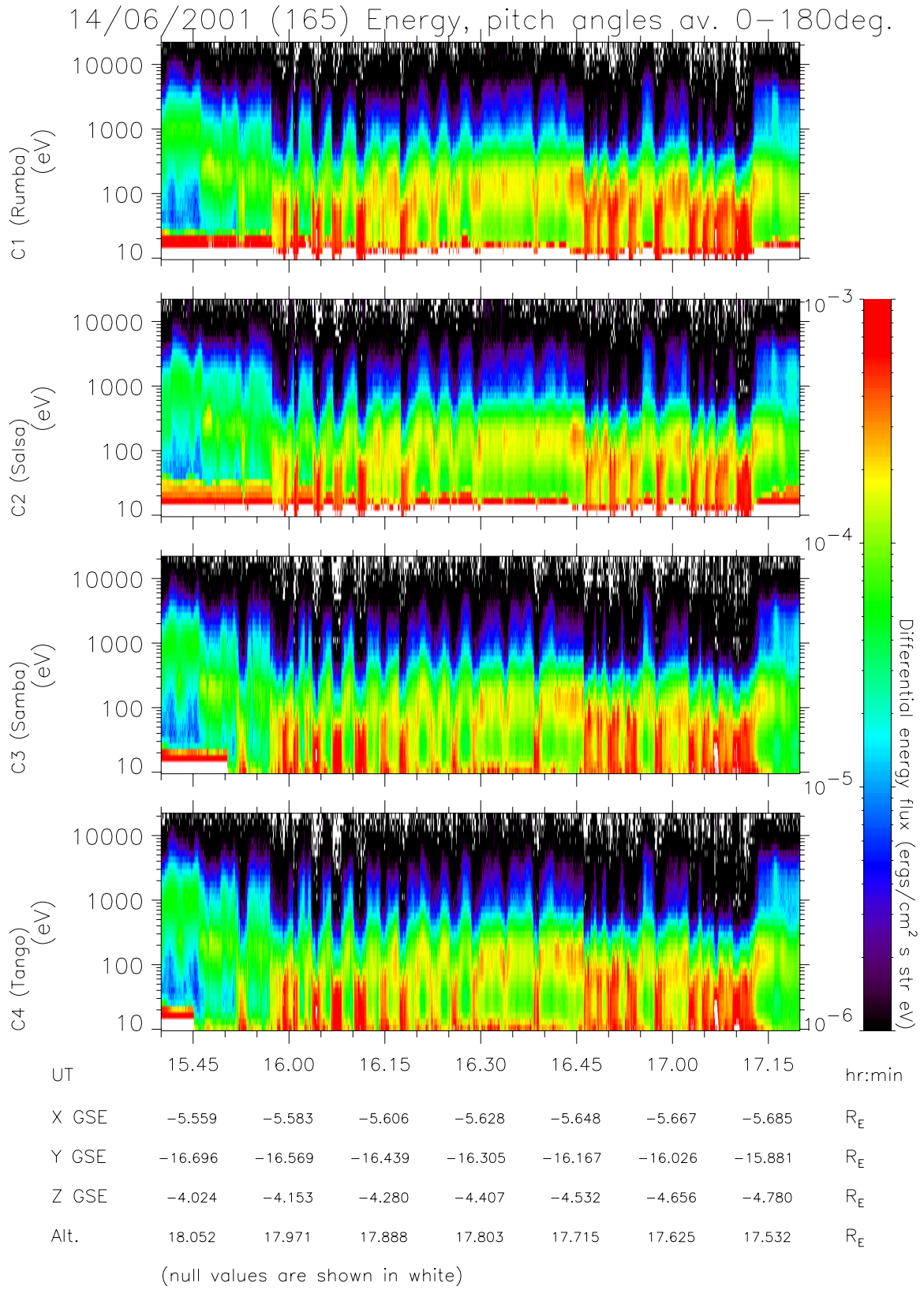

Fig. 1. Energy-time spectrograms of the electron populations observed by the 4 Cluster spacecraft between 15:40 UT and 17:20 UT on 14 June 2001. The data shown in each panel represents a direction-averaged composite of the differential energy fluxes recorded by the two PEACE sensors on each spacecraft, such that the full energy range is $10 \mathrm{eV}$ to $32 \mathrm{keV}$. The colour bar for the flux levels is shown on the right, while the ephemera data for the reference spacecraft (Cluster 3) is shown at the bottom of the figure. During the period shown, the quartet was located on the dawn flank of the magnetosphere. The data indicate that all 4 spacecraft underwent a series of transitions between the magnetosheath (which exhibits intense differential energy fluxes peaked at energies of a few $10 \mathrm{~s}$ of eV) and the magnetosphere or magnetopause boundary layer (where the differential energy fluxes are less intense but peaked at energies of 100-200 eV). Note that the transitions between these two populations are rather periodic over this period, suggesting that the Cluster quartet is observing the effects of a magnetopause boundary wave during this period.

of each spacecraft between the magnetosphere and the magnetosheath to calculate the speed and direction of those parts of the surface wave which cross the Cluster quartet. In the next section, we briefly discuss the instrumentation on board each of the Cluster spacecraft which is utilized in this study. In Sect. 3, we describe the Cluster observations made dur- ing the period $\sim 16: 00-17: 00$ UT on 14 June 2001, which indicate that the quartet made a large number of somewhat regularly-spaced crossings between the magnetosphere and the magnetosheath. In Sect. 4, we discuss the implications of these new results before drawing our conclusions in Sect. 5. 


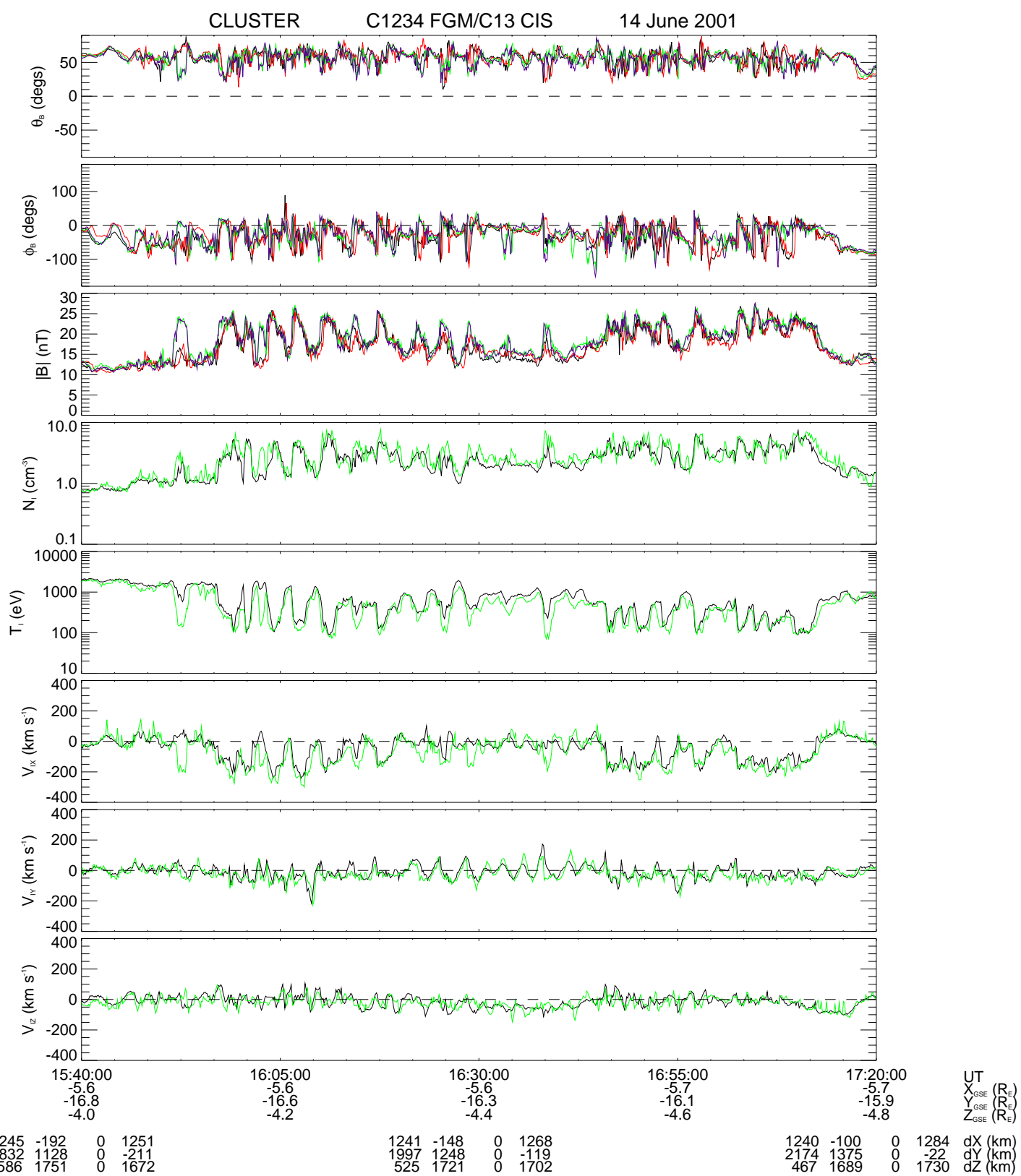

Fig. 2. Magnetic field and ion plasma data from the FGM and CIS instruments, respectively. Data from Cluster 1 is shown as the black traces, Cluster 2 in red, Cluster 3 in green and Cluster 4 in magenta. The top 3 panels contain the 4 spacecraft FGM data, showing the elevation angle, $\theta_{B}$, the azimuth angle, $\phi_{B}$, and strength $|B|$ of the magnetic field vector. The lower 5 panels show the ion density, temperature, and 3 components of the flow velocity observed by spacecraft 1 and 3 . The cold dense plasma observed when the spacecraft are in the magnetosheath is associated with field strengths of $|B| \sim 25 \mathrm{nT}$, while inside the magnetosphere the field strength of $|B| \sim 15 \mathrm{nT}$. The field direction on the two sides of the boundary is similar and points strongly northward, with only small deflections in field direction at the transitions. Inside the magnetosphere the flow velocities are low, $|V|<\mathrm{a}$ few tens of $\mathrm{km} \mathrm{s}^{-1}$, while in the magnetosheath an anti-sunward $\left(-V_{X}\right)$ flow of $100-200 \mathrm{~km} \mathrm{~s}^{-1}$ is observed. The y-component of the flow shows an oscillation about $V_{Y}=0$.

\section{Instrumentation}

This study uses electron measurements from the PEACE (Plasma Electron And Current Experiment) instruments (Johnstone et al., 1997; Owen et al., 2001) on the 4 Cluster spacecraft, together with supporting observations of the magnetic field by the FGM (Flux Gate Magnetometer) (Balogh et al., 2001) and CIS (Cluster Ion Spectrometer) (Rème et al., 2001) instruments. The PEACE instrument consists of two hemispherical electrostatic analyzers on each spacecraft which measure the three-dimensional velocity distribution of electrons with energies between $\sim 1 \mathrm{eV}$ and $26 \mathrm{keV}$. The two sensors on each spacecraft measure a different, but overlapping, part of the full energy range. On 14 June 2001 
the energy of electrons measured by the low-energy electron analyzer (LEEA) covered the range $\sim 1 \mathrm{eV}$ to $1 \mathrm{keV}$, while the high-energy electron analyzer (HEEA) covered the range $\sim 40 \mathrm{eV}$ to $26 \mathrm{keV}$. The FGM instrument consists of two triaxial sensors capable of sampling up to 67 vectors/s. In this study we use spin resolution $(4 \mathrm{~s})$ magnetic field data. The CIS instrument provides information on the ion populations from thermal energies to about $40 \mathrm{keV} / \mathrm{e}$.

\section{Observations}

On 14 June 2001 (day 165) the 4 Cluster spacecraft made multiple encounters with the dawnside flank magnetopause between $\sim 16: 00$ and $\sim$ 17:00 UT. At 16:30 UT the Cluster centroid was located at position $(-5.5,-16.3,-4.2) R_{E}$ (Earth radii) in the Geocentric Solar Ecliptic (GSE) coordinate system. Spectrograms summarizing the PEACE data taken during this period are shown in Fig. 1. The 4 panels in this figure show an energy-time spectrogram of the PEACE data recorded by each of the 4 spacecraft between 15:40 UT and 17:20 UT on this day. Each panel indicates the differential energy flux observed between $10 \mathrm{eV}$ and $26 \mathrm{keV}$, and thus is a composite of data obtained by both of the PEACE sensors on each spacecraft. These summary data consist of a direction-averaged electron flux derived on the ground from a 2-D sample of the full 3-D distribution that is returned from the spacecraft. This 2-D cut is taken from the plane that contains both the spacecraft spin vector and the instantaneous magnetic field direction. An appropriate transformation of this sample thus provides a measure of the pitch-angle distribution of the ambient electron population, under the assumption that this is gyrotropic. Ephemera data for the reference spacecraft (Cluster 3) are given at the bottom of the plot. Crossings of the magnetopause by each of the 4 spacecraft can be identified in this figure by both the changes in the energy of the peak flux and the flux levels of the observed electrons as the spacecraft moves between the magnetosheath and magnetosphere. Specifically, observations of differential energy fluxes of the order of $10^{-3} \mathrm{ergs}\left(\mathrm{cm}^{2} \mathrm{~s} \mathrm{streV}\right)^{-1}$ peaked between 10 and $100 \mathrm{eV}$, indicating that the spacecraft were sampling magnetosheath plasma, while maxima in the fluxes located between 100 and $300 \mathrm{eV}$ represent observations of boundary layer plasma inside the magnetosphere. The high fluxes of electrons at energies of $\sim 10 \mathrm{eV}$ and below are most likely a result of photo-electrons of spacecraft origin entering the instrument apertures and should be ignored.

The magnetic field data from the FGM instrument on each of the 4 spacecraft and ion plasma data from the CIS instruments on spacecraft 1 and 3 are shown in Fig. 2. (Black traces represent $\mathrm{C} 1$ data, red for $\mathrm{C} 2$, green for $\mathrm{C} 3$ and magenta for C4.) The top 3 panels show the GSE elevation angle, $\theta_{B}$, the azimuth angle, $\phi_{B}$, of the magnetic field direction, and the magnitude of the magnetic field. In this representation, $\theta_{B}=90^{\circ}$ represents a magnetic field pointing directly northward, $\theta_{B}=0^{\circ}$ represents a magnetic field lying within the ecliptic plane, while $\phi_{B}=0^{\circ}$ indicates a field with a sun- ward component, $\phi_{B}=90^{\circ}$ a field with a duskward component. These 3 panels show that for the most part, the 4 spacecraft observe similar magnetic fluctuations throughout this period. The transitions from the magnetosheath into the magnetosphere (and vice versa) are marked by a change in the magnetic field strength, with the magnetosheath side having an average $|B| \sim 25 \mathrm{nT}$, while that in the magnetosphere has a somewhat lower average value, $|B| \sim 15 \mathrm{nT}$. However, the field direction is less variable between the two regions, and remains predominantly northward and dawnward $\left(\theta_{B} \sim 60^{\circ}\right.$, $\phi_{B}=-50^{\circ}$ on average) on both sides. Short duration shifts in the direction of the magnetic field tend to occur at the boundary between the two plasma regions.

The lower 5 panels of Fig. 2 show the density, temperature, and 3 components of the velocity of the ions detected by the CIS instruments on Clusters 1 and 3. In these panels, the transition across the boundary is marked by a change from a cold, dense plasma in the magnetosheath to a hotter, more rarified plasma inside the magnetosphere. In the latter region the plasma flow speeds are very low $(V<$ a few tens of $\mathrm{km} \mathrm{s}^{-1}$ ), while in the magnetosheath anti-sunward flows at speeds of $100-200 \mathrm{~km} \mathrm{~s}^{-1}$ are observed. Note, also, that the y-component of the flow shows an oscillation about $V_{Y}=0$.

A more detailed set of PEACE spectrograms, for the 4min period 16:04-16:08 UT, is shown in Fig. 3, in the same format as Fig. 1. These cover a transition of the 4 spacecraft from the magnetosheath and into the magnetosphere at 16:05:15 UT, followed by a return to the magnetosheath by all 4 spacecraft at $\sim 16: 06: 30$ UT. It is immediately apparent from this figure that the exit from and re-entry to the magnetosheath were observed at different times at each of the four spacecraft. In addition, the nature of the entry and exit are qualitatively different. The exit from the magnetosheath observed by each spacecraft consists of a gradual reduction in the differential energy flux and a gradual increase in the electron temperature. While located inside the magnetosphere/boundary layer, $\mathrm{C} 3$ and $\mathrm{C} 4$ observe noticeable variations in the electron fluxes. In contrast, the return transitions are generally more abrupt, with a discontinuous change in the electron population from that characteristic of the boundary layer to that characteristic of the magnetosheath. The two sets of magnetopause (MP) crossings shown in this figure are rather typical of all the crossings observed during this period.

The relatively close spacing $(\sim 2000 \mathrm{~km})$ and tetrahedral configuration of the Cluster quartet allows, for the first time, for an analysis of the 3-dimensional motion of the boundaries encountered by the spacecraft. In order to achieve this, a method which consistently identifies the times at which each spacecraft crosses a given boundary must be developed. In principle, the boundary timings can be assessed from any of the data sets which are available from 4 spacecraft. Sophisticated boundary analysis methods have been developed for use with the FGM data (e.g. Dunlop and Woodward, 1998). These methods incorporate single spacecraft assessment of the boundary orientation through minimum variance analysis, in order to check the planarity of the boundary. However, the magnetic fields on either side of the magnetopause 
boundary observed in the period described above are largely parallel. For this reason, the minimum variance analysis does not return a meaningful result for many of the boundary crossings observed during this period. The observed boundaries are predominantly plasma boundaries, and thus, in this study, we assess the boundary timings using the PEACE observations.

Once the timings of the boundary crossings at each spacecraft have been determined, this information can be used to deduce the motion of any given boundary, as described, for example, in Owen et al. (2001). In this method we must assume that the boundary is close to planar on the scale size of the spacecraft separation, and moves with constant velocity. The differences in the boundary observation times at each spacecraft position can then be used to calculate the speed and direction of motion normal to the boundary surface. As noted above, the nature of the magnetopause boundary crossings in the PEACE data is somewhat variable. However, to quantify the timings, the boundary crossings by each spacecraft must be consistently identified within the data set. To do this, we use the bulk moments of the electron population calculated on board each spacecraft. In particular, we identify the local maxima in the time rate of change of electron moments during the large changes that occur in these data at each boundary. Comparison of the results of this approach applied to both the density and scalar temperature moments revealed that the magnetopause crossings could be most readily identified from the gradients in the temperature moments from each spacecraft. It should be noted that the technique employed here to determine boundary-crossing times is adopted principally as a method whereby some aspect of the transition from the magnetosphere to the magnetosheath can be consistently identified within the PEACE data set. Since the transition from the magnetosphere to the magnetosheath must involve crossing the magnetopause, we arbitrarily chose to associate this temperature gradient maximum coincident with a large total change in temperature with the instant that each spacecraft makes a crossing of the magnetopause. We assume that any errors in the timing between this temperature gradient maximum and the real magnetopause crossing are systematic and identical at each spacecraft, such that the timing analysis results are unaffected.

Figure 4 shows the temperature moment changes during the two magnetopause crossings previously shown in Fig. 3 above. The dashed line in this figure represents the effective temperature of the electrons determined by taking a moment summation of electron populations within the HEEA $(40 \mathrm{eV}$ to $26 \mathrm{keV})$ range, while the solid line indicates the magnitude of the gradient in this quantity. Note that for the most part, the large temperature changes are associated with a clear peak in the gradient. In the C3 data at 16:05:45 UT there is a less clear association, and in this case the earlier peak in the gradient is chosen as it coincided with a larger total change in temperature than the later peak. Using the times identified as the peak gradient on each spacecraft, we can determine the motion of the boundaries as outlined above. For the case shown in Fig. 3, we find that during the transition from the magnetosheath into the magnetosphere observed at $\sim 16: 05: 30$ UT the timings are consistent with a boundary motion in the direction $(-0.53,-0.58,0.62)$ in GSE coordinates and at a speed of $118 \mathrm{~km} \mathrm{~s}^{-1}$ (i.e. GSE velocity components $\left.(-62,-68,73) \mathrm{km} \mathrm{s}^{-1}\right)$. Conversely, during the return transition from magnetosphere to magnetosheath at $\sim 16: 06: 40 \mathrm{UT}$, the timings are consistent with a boundary motion in the GSE direction $(-0.69,0.46,0.56)$ at a speed of $82 \mathrm{~km} \mathrm{~s}^{-1}$. The corresponding GSE velocity components are $(-57,38,46) \mathrm{km} \mathrm{s}^{-1}$. Note that the boundary normals determined here from the Cluster data can be directly compared to the normal calculated on the basis of the Fairfield (1971) model magnetopause scaled to the spacecraft position. This is calculated to be $(0.32,-0.92,-0.23)$ in this coordinate system. Note, also, that although a minimum variance analysis of FGM data does not return a meaningful determination of the boundary normal at each spacecraft for many of the observed crossings, it can be used as a successful check on the 4-spacecraft timing result for those crossings that it is successful. For the first crossing evident in Fig. 3, the minimum variance analysis of the corresponding magnetic field (not shown) fails. However, during the second crossing there are sufficient variances in the magnetic field to determine an MVA normal at the 4 spacecraft. In each case, the MVA normal is within $15^{\circ}$ of that determined by the 4-spacecraft method, thus validating the results of the latter method.

A total of 21 magnetosheath exits and 23 entries similar to those described above could be identified in the PEACE data during the period 15:55-17:15 UT and these were analyzed using the above technique. The results of the boundary motion calculations for these crossings are summarized in Fig. 5, which is comprised of three panels showing, from top to bottom, the boundary velocities in the $X-Y, X-Z$ and $Y-Z$ GSE planes. Each panel includes the projection of the orbit trajectory of the reference spacecraft (Cluster 3 ) in the relevant plane (black line), together with the locus of the intersection of that plane with the model magnetopause derived from Fairfield (1971) scaled to the spacecraft position (green line). The boundary velocities are shown as vectors in each panel, with the speed of the boundary in that plane being proportional to the length of the vector drawn and the direction in each case, indicated from the point on the spacecraft trajectory at which the boundary motion was determined. The length of a $100 \mathrm{~km} \mathrm{~s}^{-1}$ velocity vector is shown to the right of each panel for comparison. Boundary motions corresponding to the transition of the spacecraft from the magnetosheath into the magnetosphere are shown as blue vectors, while transitions from magnetosphere to the magnetosheath are shown as red vectors. The differences between the magnetosphere entry and exit vectors are most apparent in the $Y-Z$ plane (lower panel), where the entries (blue vectors) generally have a dawnward component, while the exits (red vectors) generally have a duskward component. From the second panel, it can be seen that almost all the velocity vectors have both an anti-sunward and a northward component to their direction, and that the exits (red vectors) tend to have a larger 


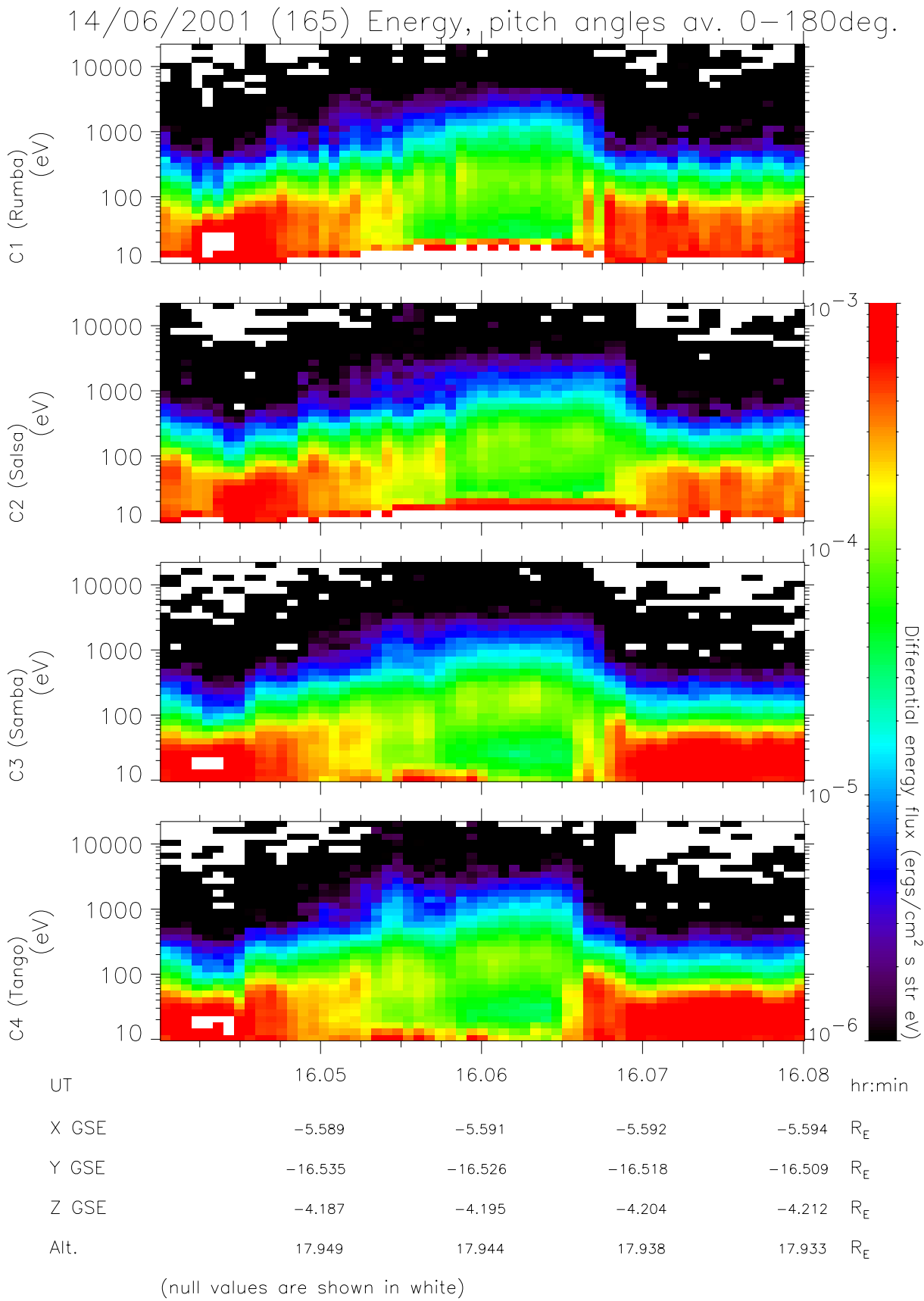

Fig. 3. Energy-time spectrograms of the electron populations observed by the 4 Cluster spacecraft between 16:04 UT and 16:08 UT on 14 June 2001. The figure is in the same format as Fig. 1, but covers a shorter time period, during which the Cluster quartet made a single transition from the magnetosheath into the boundary layer ( 16:05:15 UT) and then back into the magnetosheath $(\sim 16: 06: 30$ UT). Note that the entries by all 4 spacecraft into the magnetosphere at $\sim 16: 05: 15$ UT appear to be a more gradual transition than the exits at $\sim 16: 06: 30$ UT, which show a much more discontinuous change in the electron fluxes. This single entry-exit transition has characteristics that are largely representative of all the other transitions evident in Fig. 1.

northern component than the entries. The average over all the velocity magnitudes is $\sim 100 \mathrm{~km} \mathrm{~s}^{-1}$, with the average magnitude of the exit velocities being $\sim 120 \mathrm{~km} \mathrm{~s}^{-1}$ and the entry velocities $\sim 80 \mathrm{~km} \mathrm{~s}^{-1}$. The angles between the boundary motion normals determined from the inter-spacecraft timings and the model MP normal $((0.32,-0.92,-0.23)$ in GSE co- ordinates) are shown in the upper two panels of Fig. 6. The top panel of this figure shows these angles as a function of time of observation of the boundary during exits into the magnetosheath, while the lower panel shows the angles as the spacecraft enter back into the magnetosphere. Note that for an in-out breathing motion of the entire magnetopause, 


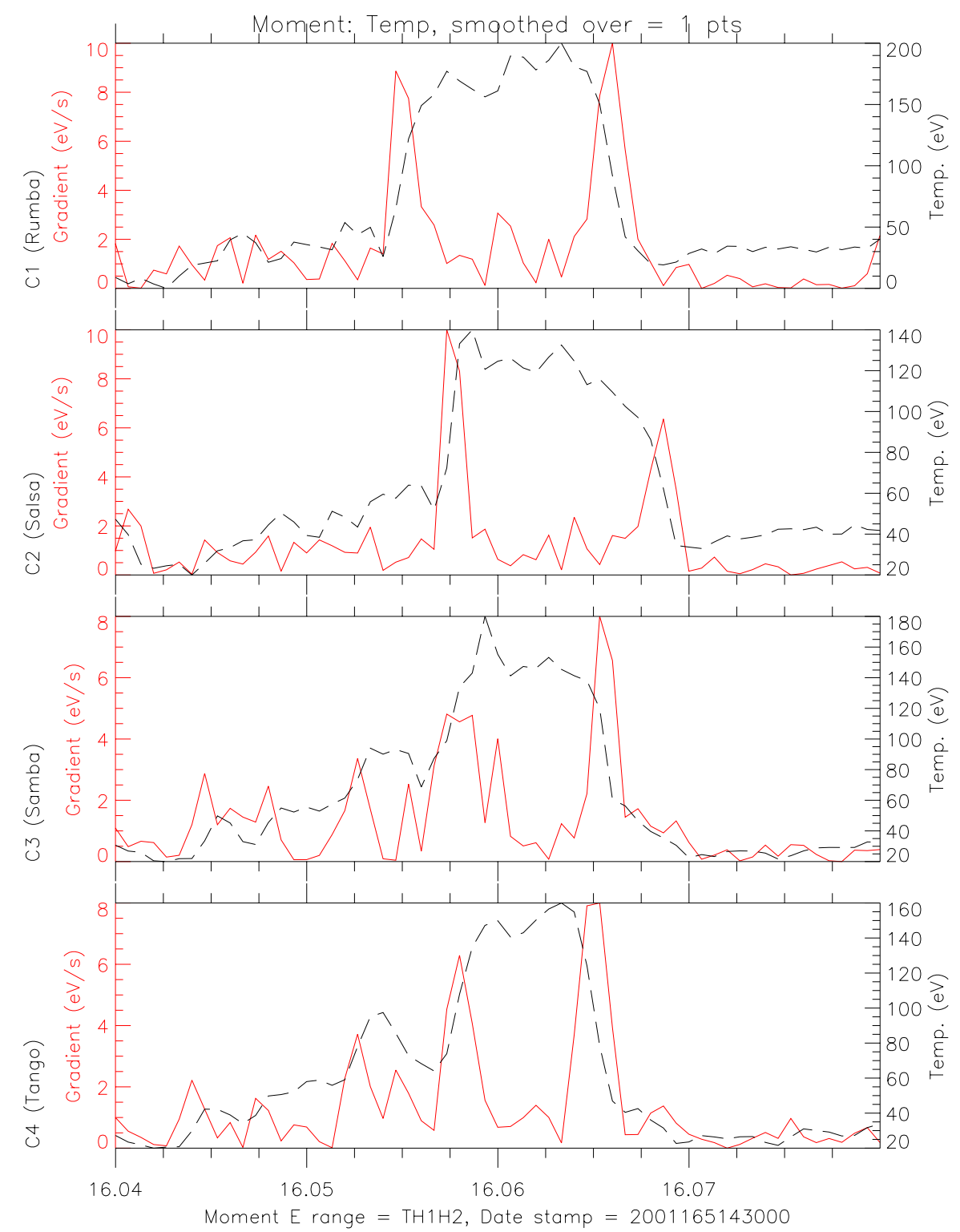

Fig. 4. Electron scalar temperature variations (dashed line, right-hand scale) observed by the 4 Cluster spacecraft between 16:04 UT and 16:08 UT on 14 June 2001 (c.f. Fig. 3). The transitions between the cool $(\sim 30-40 \mathrm{eV})$ magnetosheath and warmer boundary layer ( $>100 \mathrm{eV})$ plasmas are evident in each panel. The solid lines (related to the left-hand scale) indicate the magnitude of the gradient in these temperature profiles between each measurement. Relatively clear peaks occur in this parameter for each major transition in plasma temperature. These peaks are used as a measure of the time that the boundary between the two plasma populations crosses each of the 4 spacecraft, and thus to determine the orientation and speed of this boundary (see text).

these angles would be $0^{\circ}$ for entries into the magnetosphere, associated with dawnward motion of the boundary, and $180^{\circ}$ as the boundary moves duskward to return the spacecraft to the magnetosheath. The dashed lines in each panel represent the average normal angle for all the magnetospheric exit and entry events, respectively. Note that the angles associated with entries to the magnetosphere are more variable than the angles during exit. The mean normal angle during entries is $68^{\circ}$ away from the model magnetopause normal, i.e. only $22^{\circ}$ out of the plane of the model magnetopause itself and pointing into the magnetosheath (i.e. somewhat dawnward).
In contrast, the mean angle during exits is $138^{\circ}$, or $42^{\circ}$ away from the model magnetopause plane and pointing into the magnetosphere.

\section{Discussion}

The observations of boundary motion presented above clearly show differences in velocity and angles between exit and entry transitions, which are generally consistent with a boundary wave. Note that the $y$-component of the boundary velocities reverses between the inbound and outbound transitions shown in Fig. 4, as might be expected during a 

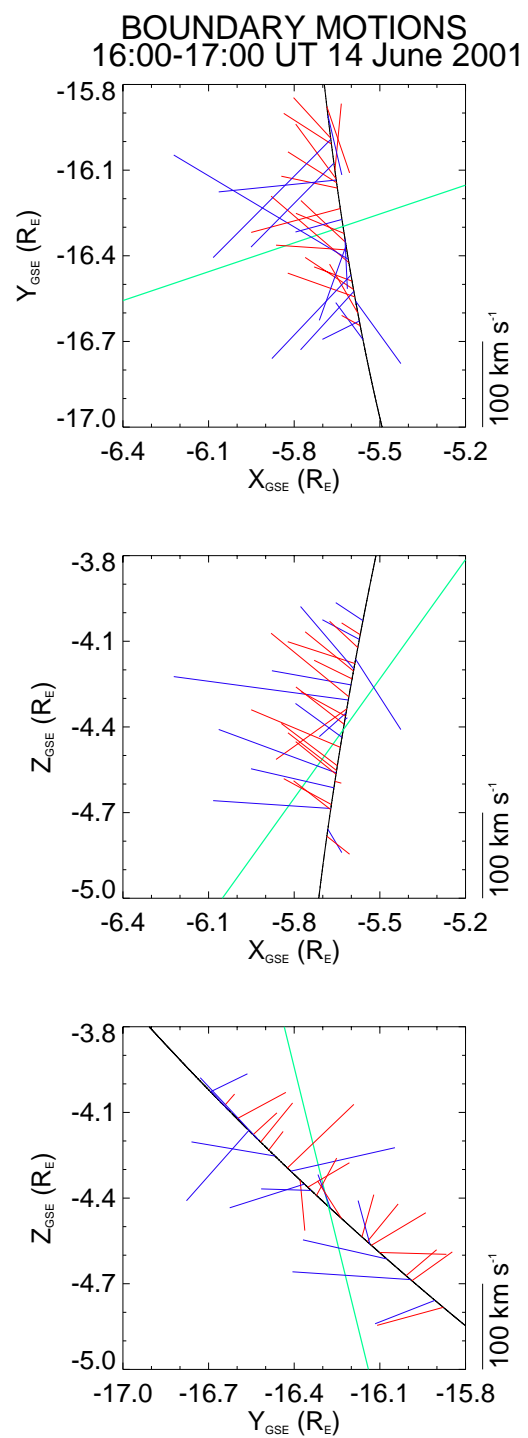

Fig. 5. Results of the boundary motion analysis for each of the transitions evident in Fig. 1. The 3 panels show the motion of the boundary projected onto each of the $X-Y, X-Z$ and $Y-Z$ GSE planes. The intersection of the Fairfield (1971) model magnetopause surface (scaled to the spacecraft location at 16:30 UT) with each plane is shown as the green curve in each panel. The spacecraft trajectory projected onto each plane is shown as the black trace. The projection of vectors representing the speed and normal direction of motion of each magnetosheath-magnetosphere boundary in each plane are shown extending from the point on the orbit trajectory corresponding to their time of observation. Vectors determined for transitions from magnetosheath to magnetosphere are shown in red, while those for transitions in the opposite sense are shown in blue. The length of a vector representing $100 \mathrm{~km} \mathrm{~s}^{-1}$ is indicated at the bottom of the plot. From these results it is clear that most boundary motions involve motion in the negative $X_{\mathrm{GSE}}$ direction, indicating a tailward propagation of the wavetrain. In the $Y-Z$ plane (lower panel) it is also clear that most boundaries move in the general directions expected for inbound/outbound crossings of the magnetopause, consistent with motions of the magnetopause driven by a set of boundary waves moving across the spacecraft locations. large-scale breathing motion of the magnetopause. Indeed, since the Cluster quartet is located close to the equatorial flank of the magnetosphere, any large-scale inward/outward breathing of the magnetopause should manifest itself as a predominant positive/negative $y$-component of the boundary velocity. This oscillation is also seen in the $V_{Y}$ component of the ion velocity presented in Fig. 2. Clearly, however, both the inbound and outbound boundary motions, consistent with the data shown in Fig. 4, have significant negative $x$ - and positive $z$-components to their motion, indicating a boundary wave structure with wave fronts moving along the magnetopause in these directions. An anti-sunward (i.e. negative $\mathrm{x}$-directed) component of motion is perhaps not surprising for boundary waves that may be driven by the magnetosheath flow. However, the strong, positive $z$-component to the motion is perhaps unexpected, especially as the Cluster quartet is located slightly below the ecliptic plane where models of the exterior magnetosheath flow (e.g. Spreiter and Stahara, 1980) indicate a small component of flow in the negative $z$ direction. Indeed, no significant $z$-component of the flow velocity is observed by the CIS instrument (Fig. 2) during the period under consideration.

The results from the single wave period shown in Fig. 4 are confirmed as representative of the whole period in Figs. 5 and 6 , which show that on both entry to and exit from the magnetosphere, the local magnetopause is tilted quite sharply away from its undisturbed orientation (as based on the Fairfield, 1971 model). In addition, these figures clearly show that during boundary encounters associated with entries into the magnetosphere, the boundary is, on average, tilted further away from its undisturbed orientation than during the returns to the magnetosheath. A sketch illustrating a possible form of this wave is given in Fig. 7. The entries into the magnetosphere discussed above have been drawn to correspond to the leading edges of the wave, while the exits to the magnetosheath correspond to the trailing edges. The entry and exit angles shown in the sketch are based on the mean angles determined from the data shown in Fig. 6. The wave faces thus appear to be steepest on the leading edge (i.e. those associated with entries to the magnetosphere which are inclined into the anti-sunward direction of travel). This observed steepening of the leading edge of the wave, with a shallower trailing edge is consistent with KH theory (Chandrasekhar, 1961) and MHD simulations of KH waves (Miura, 1990). However, some previous observations are in contrast to this observation. In particular, the two-point study using ISEE 1 and 2 by Chen et al. (1993) concluded that the trailing edge of magnetopause surface waves was steeper than the leading edge. We note that the difference between the observed normal angles and the model magnetopause boundary normal is clearly a function of the appropriateness of the model in representing the undisturbed magnetopause at this time. However, we believe that it is unlikely that the model magnetopause used here is inaccurate by more than $\sim 10^{\circ}$ or so. Hence, although the absolute departures of the observed normals from those of the model may change, the conclusion that the angles determined from the 4-spacecraft timing 

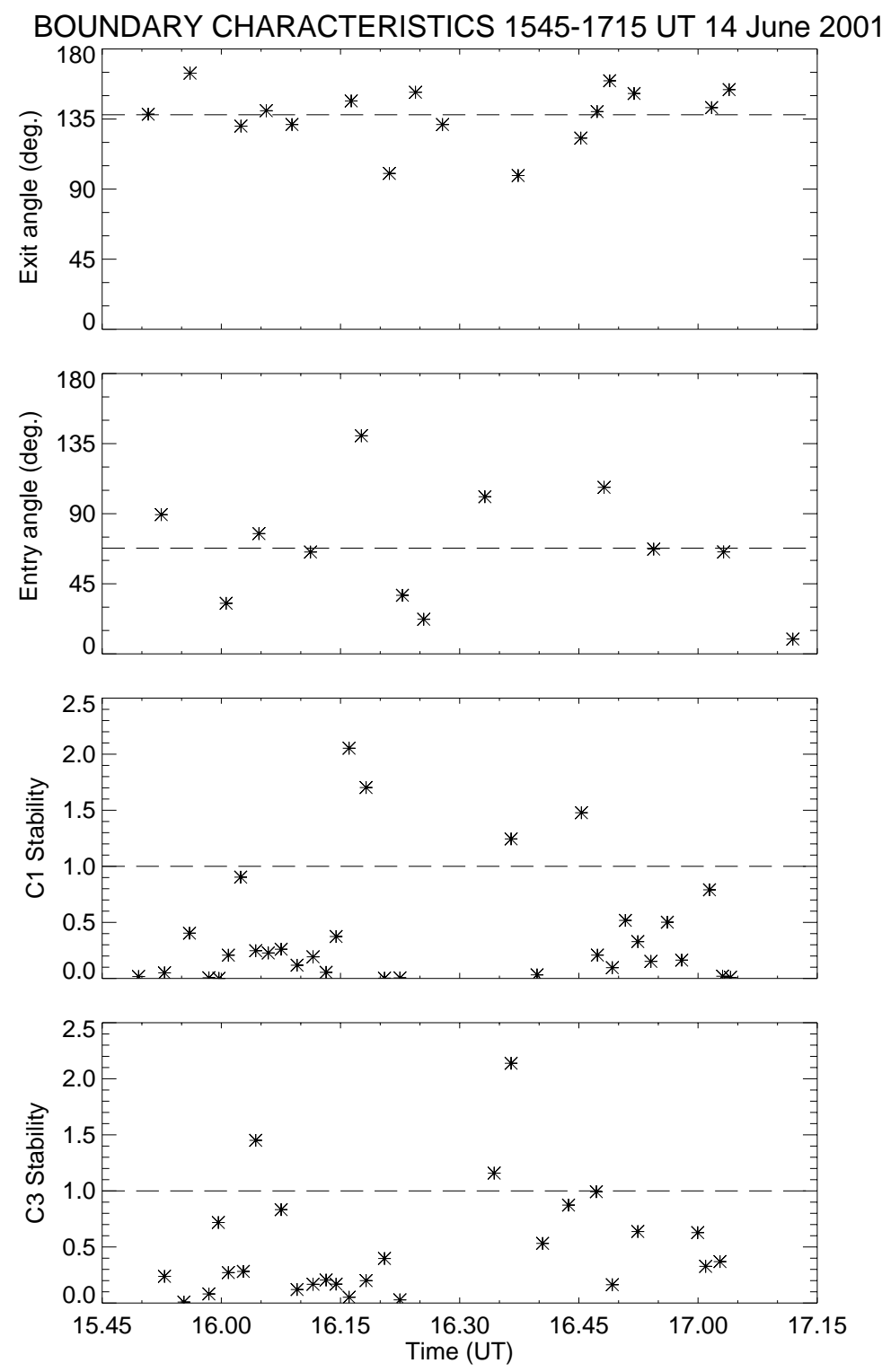

Fig. 6. Upper 2 panels: Plots of the angles between the boundary normals determined from the 4-spacecraft measurements and the Fairfield (1971) model magnetopause normal as a function of time of observation of each boundary. The upper panel shows the angles determined during transitions from the magnetosphere into the magnetosheath, while the lower panel shows those angles determined during transitions in the opposite sense. The dashed line in each panel represents the mean angle for the appropriate data set. Note that the wavefronts during the latter transition are, on average, $79^{\circ}$ away from the direction expected on the basis of the model magnetopause. In contrast, the transitions from magnetosphere into the magnetosheath are only $34^{\circ}$ away from the expected direction (along the inward pointing model normal). Thus, the wavefronts associated with the former transition appear to be significantly steeper inclined, on average, compared to the latter. Lower Panels: Results of a Kelvin-Helmholtz stability analysis (c.f. Eq. (1)) for the magnetopause boundary based on data from Clusters 1 and 3. The vertical axes of these panels show the ratio $R$ of the two terms on either side of the inequality symbol in Eq. (1), such that values of $R>1$ (i.e. above the dashed line in each panel) indicate the magnetopause to be Kelvin-Helmholtz unstable. For most of the period under discussion, it appears that the magnetopause is stable to this mechanism. However, the brief periods for which instability is indicated appear to correspond to times in which the angles of the observed wavefronts (upper 2 panels) are furthest from the expectations for an undisturbed model magnetopause. This indicates that the wavefronts are steepest during these times.

analysis as the spacecraft exits the magnetosheath are steeper than the entry angles is most likely appropriate. In addition, we note that our results are consistent with those of some other studies (e.g. Fairfield et al., 2000).
To test whether the magnetopause is unstable to the growth of Kelvin-Helmholtz waves, we can attempt to evaluate the inequality given in Eq. (1) above for each of the boundary crossings. If the inequality is satisfied the magnetopause is Kelvin-Helmholtz unstable. For this purpose, we use the ion 
plasma velocities $\boldsymbol{V}_{\mathbf{1}}$ and $\boldsymbol{V}_{\mathbf{2}}$ on either side of a boundary crossing which are provided by the CIS instruments, the magnetic fields $\boldsymbol{B}_{\mathbf{1}}$ and $\boldsymbol{B}_{\mathbf{2}}$ provided by the FGM instrument and the electron number density $n$ from PEACE. In order to make an assessment of $\boldsymbol{k}$, the unit wave vector, we rotate the mean observed boundary normal, $\boldsymbol{n}_{B D Y}$, (for pairs of magnetosphere entries and exits) into the plane of the model magnetopause via $\boldsymbol{k}=\boldsymbol{n}_{M P} \times\left(\boldsymbol{n}_{B D Y} \times \boldsymbol{n}_{M P}\right)$, where $\boldsymbol{n}_{M P}$ is the model magnetopause normal. The results of the instability analysis for $\mathrm{C} 1$ and $\mathrm{C} 3$ are shown in the lower panels of Fig. 6. The CIS ion data from $\mathrm{C} 2$ and $\mathrm{C} 4$ were not available during this interval and thus, we are unable to perform this analysis on the observations from these spacecraft. In the lower panel, we show the ratio, $R$, of the terms on each side of the inequality sign in Eq. (1), such that a value of $R$ larger than unity suggests an unstable boundary. For most of the interval, the analysis indicates that the waves are KelvinHelmholtz stable $(R<1)$. However, when comparing these results to the wave exit and entry angles in the upper panels, we find that instances where the observed data are consistent with the magnetopause being Kelvin-Helmholtz unstable $(R>1)$ generally occur in association with increases in the wave exit angle, i.e. a steepening of the leading edge of the wave. The Kelvin-Helmholtz instability is predicted to cause such a steepening of the leading edge of surface waves (Miura, 1990), consistent with our observations. However, waves generated by means other than the Kelvin-Helmholtz mechanism might also be expected to show a similar trend between their leading and trailing edges, such that this alone does not provide a conclusive identification of the wave driving mechanism. Although the above analysis is inconclusive in this case, it is informative to consider the general orientation of the boundary wave fronts to the magnetosheath field and flow directions. As mentioned above, the wave fronts generally appear to have a positive $z$-component to their motion, despite the location some $4.5 R_{E}$ south of the ecliptic plane. During this period, the average magnetic field observed while the spacecraft are in the magnetosheath is $(11.5$, $-3.4,17.7) \mathrm{nT}$, while in the magnetosphere the average field $(6.4,-3.9,14.2) \mathrm{nT}$. The average fields on either side of the magnetopause boundary are thus close to parallel, separated in direction by only about $10^{\circ}$. Moreover, the fields point predominantly in the positive $X$ - and positive $Z$-directions, which is approximately perpendicular to the direction of motion of the wave fronts inferred above. For this direction of motion, therefore, the magnetic fields on either side of the boundary lies along the crests and troughs of the wave, as also indicated in the sketch in Fig. 7. Hence, the wave appears to be moving in the one direction in which the magnetic fields on either side of the magnetopause do not need to be sharply bent in order to accommodate the wave, and that most energy is, therefore, available for the wave growth. This can also be seen mathematically in Eq. (1), in which the terms $\boldsymbol{k} . \boldsymbol{B}$ are smallest, and thus the inequality most likely to be satisfied, if the wave vector $\boldsymbol{k}$ is perpendicular to the magnetic field $\boldsymbol{B}$. This appears to be the case, at least on average, in the event presented here.

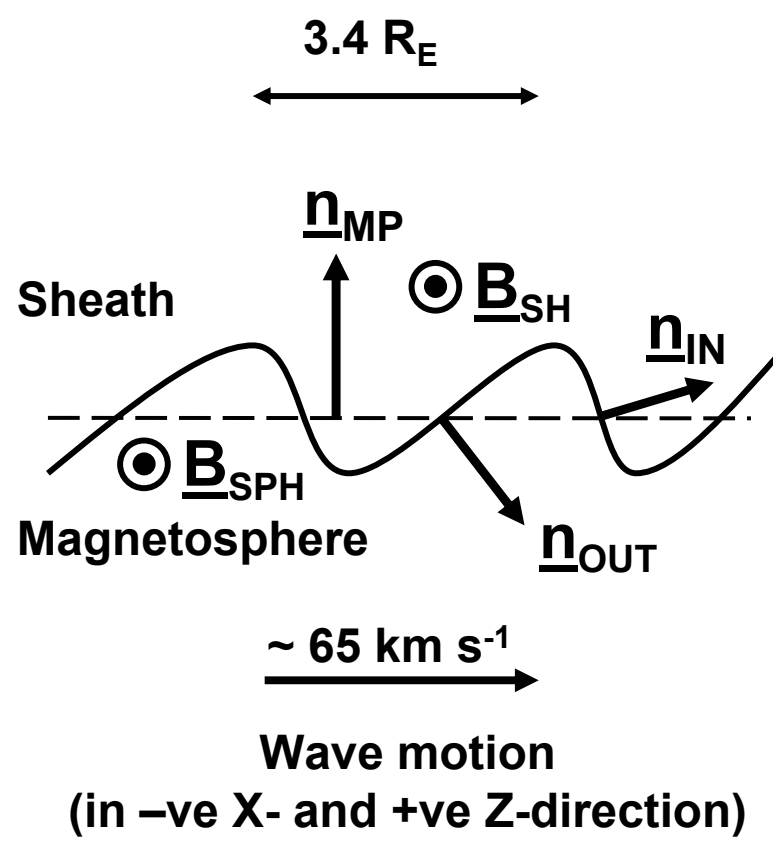

Fig. 7. Sketch illustrating the average structure of the magnetopause surface wave consistent with the results of the 4-spacecraft timing analysis. These results suggest that the leading edge of the waves, associated with transitions of the spacecraft from magnetosheath to magnetosphere, are inclined from the expected orientation of the undisturbed magnetopause by a larger angle, on average, than the trailing edge associated with transitions in the opposite sense. In addition, the direction of propagation of the waves, in the negative GSE $X$ - and positive $Z$-directions, is close to perpendicular to the magnetic fields on either side of the boundary. This suggests that the wave growth and propagation occurs preferentially in the direction in which the wave does not have to bend the surrounding lines of magnetic force.

Finally, the wavelength of these surface waves can be estimated by using the mean velocity of the waves across the magnetopause $\left(\sim 65 \mathrm{~km} \mathrm{~s}^{-1}\right)$ and the time differences between pairs of boundary crossings. In this manner, we find the mean wavelength is approximately $3.4 R_{E}$.

\section{Conclusions}

Surface waves are common phenomena around the lowlatitude flanks of the magnetopause (Kivelson and Chen, 1995; Seon et al., 1995). We have presented an example of such waves observed by the 4-spacecraft Cluster mission, located on the dawn flank, on 14 June 2001. During a period of $\sim 1 \mathrm{~h}$, the PEACE instrument on each spacecraft observed a large number of quasi-regular transitions between an electron plasma population with characteristics typical of that of the magnetosheath and a more diffuse population of higher energy which is typical of the flank magnetosphere/boundary layer. The timings of each of these transitions at each spacecraft have been determined by identifying the times of maximum gradient in the electron temperature moment. Differences in the transition times at each of the 
4 spacecraft have been used to determine the local orientation and speed of the associated magnetopause boundary as it moves back and forth over the spacecraft location. These orientations can be compared with the expectations for the average magnetopause orientation determined from model fits to historical data (e.g. Fairfield, 1971). This comparison reveals that both the inbound and outbound crossings of the magnetopause are significantly deflected from the expected orientation, in the senses that are indeed consistent with the passage of a boundary wave past the spacecraft location. We find also that the leading edge of the wave is significantly steeper in inclination to the model magnetopause than the trailing edge. This observation is consistent with expectations of Kelvin-Helmholtz waves (and possibly waves of other origin), but contradicts the conclusions of the 2 spacecraft study presented by Chen et al. (1993). We tested the conditions on either side of the magnetopause to determine whether they satisfied the conditions for this boundary to be Kelvin-Helmholtz unstable, based on the simple incompressible plasma model. The boundary was found to be largely stable during this period. However, we note that there is some suggestion in the results that the sub-intervals which satisfy the instability criterion are closely matched in time with the steepest boundary orientations. The 4-spacecraft timing results can also be used to determine the wavelength of the surface waves and their speed and direction of propagation across the model magnetopause surface. For this event, we find an average wavelength of $\sim 3.4 R_{E}$, and that the waves propagate at an average speed of $\sim 65 \mathrm{~km} \mathrm{~s}^{-1}$ in an anti-sunward and northward direction. The northward component to the motion is unexpected, since the spacecraft are located south of the GSE $Z=0$ plane, where the large-scale magnetosheath flow outside the magnetopause is expected to have a southward component at this location. Indeed, the ion flows observed when the spacecraft are in the magnetosheath (c.f. Fig. 2) do not show a significant northward component. However, the propagation direction is approximately perpendicular to the average external magnetic field direction, suggesting that these waves may preferentially propagate in the direction which requires no bending of magnetic lines of force. Although this observation is also consistent with waves driven by the KHI, which are expected to grow fastest when $\boldsymbol{k} . \boldsymbol{B}$ is small, it may also be the energetically most favourable conditions for the growth of other waves. Hence, although the waves show characteristics that might be expected of those driven by the KHI, we are unable at present to definitively identify them as such.

Acknowledgements. This work was funded by the UK Particle Physics and Astronomy Research Council (PPARC). In particular, CJO would like to acknowledge support via a PPARC Advanced Fellowship. We also thank Cluster Principal Investigators A. Balogh and H. Rème for use of FGM and CIS data in this study.

Topical Editor T. Pulkkinen thanks D. Fairfield and another referee for their help in evaluating this paper.

\section{References}

Axford, W. I. and Hines, C. O. : A unifying theory of high-latitude geophysical phenomena and geomagnetic storms, Can. J. Phys., 39, 1433-1464, 1961

Balogh, A., Carr, C. M., Acuna, M. H., Dunlop, M. W., Beek, T. J., Brown, P., Fornacon, K.-H., Georgescu, E., Glassmeier, K.-H., Harris, J., Musmann, G., Oddy, T., and Schwingenschuh, K.: The Cluster magnetic field investigation: overview of in-flight performance and initial results, Ann. Geophysicae, 19, 1207-1217, 2001.

Chandresekhar, S.: Hydrodynamic and Hydromagnetic Stability, Clarendon, Oxford, 1961.

Chen, S.-H. and Kivelson, M. G.: On Non-Sinusoidal Waves at the Earths Magnetopause, Geophys. Res. Lett., 20, 2699-2702, 1993.

Chen S.-H., Kivelson, M. G., Gosling, J. T., Walker, R. J., and Lazarus, A. J.: Anomalous Aspects of Magnetosheath Flow and of the Shape and Oscillations of the Magnetopause During an Interval af Strongly Northward Interplanetary Magnetic Field, J. Geophys. Res., 98, 5727-5742, 1993.

Drazin, P. G. and Reid, W. H.: Hydromagnetic Stability, Cambridge University Press, 1985.

Dungey, J. W.: Electrodynamics of the outer atmosphere, in Proceedings of the ionosphere Conference, The Physical society of London, 225, 1955.

Dungey, J. W.: Interplanetary magnetic field and the auroral zones, Phys. Rev. Lett., 6, 47-48, 1961.

Dunlop, M. W. and Woodward, T. I.: Discontinuity analysis: orientation and motion, in: Analysis methods for multispacecraft data, ISSI Scientific Report SR-001, ESA Publications, Noordwijk, The Netherlands, 271, 1998.

Fairfield, D. H.: Average and unusual locations of the Earths magnetopause and bow shock, J. Geophys. Res., 76, 6700-6716, 1971.

Fairfield, D. H., Otto, A., Mukai, T., Kokubun, S., Lepping, R. P., Steinberg, J. T., Lazarus,A. J., and Yamamoto, T.: GEOTAIL observations of the Kelvin-Helmholtz instability at the equatorial magnetotail boundary for parallel northward fields, J. Geophys. Res., 105, 21 159-21 174, 2000.

Farrugia, C. J., Gratton, F. T., Bender, L., Biernat, H. K., Erkaev, N. V., Quinn, J. M., Torbert, R. B., and Dennisenko, V.: Charts of joint Kelvin-Helmholtz and Rayleigh-Taylor instabilities at the dayside magnetopause for strongly northward interplanetary magnetic field, J. Geophys. Res., 103, 6703-6727, 1998.

Farrugia, C. T., Gratton, F. T., Contin, J., Cocheci, C. C., Arnoldy, R. L., Ogilvie, K. W., Lepping, R. P., Zastenker, G. N., Nozdrachev, M. N., Federov, A., Sauvaud, J.-A., Steinberg, J. T., and Rostoker, G.: Coordinated Wind, Interball/tail, and ground observations of Kelvin-Helmholtz waves at the near-tail, equatorial magnetopause at dusk: 11 January 1997, J. Geophys. Res., 105, 7639-7667, 2000.

Fitzenreiter, R. J. and Ogilvie, K. W.: Kelvin-Helmholtz Instability at the Magnetopause: Observations, in Physics of the Magnetopause, edited by Song, P., Sonnerup, B. U. Ö., and Thomsen, M. F., AGU monograph, 277-284, 1995.

Johnstone, A. D., Alsop, C. Burge, S., Carter, P. J., Coates, A. J., Coker, A. J., Fazakerley, A. N., Grande, M., Gowan, R. A., Gurgiolo, C., Hancock, B. K., Narheim, B., Preece, A., Sheather, P. H., Winningham, j. D., and Woodliffe, R. D.: PEACE: A plasma electron and current experiment, Space Sci. Rev., 79, 351-398, 1997. 
Kivelson, G. K. and Chen, S.-H.: The Magnetopause: Surface waves and Instabilities and their possible Dynamical Consequences, in Physics of the Magnetopause, edited by: Song, P., Sonnerup, B. U. Ö, and Thomsen, M. F., AGU monograph, 257268, 1995.

Miura, A.: Anomalous transport by magnetohydrodynamic KelvinHelmholtz instabilities in the solar wind-magnetosphere interaction, J. Geophys. Res., 89, 801-818, 1984.

Miura, A.: Kelvin Helmholtz instability for supersonic shear flow at the magnetopause boundary, Geophys. Res. Lett., 17, 749-752, 1990.

Miura, A.: Kelvin-Helmholtz Instability at the Magnetopause: Computer Simulations, in Physics of the Magnetopause, edited by Song, P., Sonnerup, B. U. Ö, and Thomsen, M. F., AGU monograph, 285-291, 1995.

Nykyri, K. and Otto, A.: Plasma transport at the magnetospheric boundary due to reconnection in Kelvin-Helmholtz vortices, Geophys. Res. Lett., 28, 3565-3568, 2001.

Ogilvie, K. W. and Fitzenreiter, R. J.: The Kelvin-Helmholtz instability at the magnetopause and the inner boundary layer surface, J. Geophys. Res., 94, 15 113-15 123, 1989.

Otto, A. and Fairfield, D. H.: Kelvin-Helmholtz instability at the magnetotail boundary: MHD simulation and comparison with GEOTAIL observations, J. Geophys. Res., 105, 21 175-21 190, 2000.

Owen, C. J., Fazakerley, A. N., Carter, P. J., Coates, A. J., Krauklis, I. C., Szita, S., Taylor, M. G. G. T., Travnicek, P., Watson, G., Wilson, R. J., Balogh, A., and Dunlop, M. W.: CLUSTER PEACE Observations of Electrons During Magnetospheric Flux Transfer Events, Ann. Geophysicae, 19, 1509-1522, 2001.

$\mathrm{Pu}$, Z.-P. and Kivelson, M. G.: Kelvin-Helmholtz instability at the magnetopause: Solution for compressible plasma, J. Geophys. Res., 88, 841-852, 1983a.
$\mathrm{Pu}$, Z.-P. and Kivelson, M. G.: Kelvin-Helmholtz instability at the magnetopause: Energy flux into the magnetosphere, J. Geophys. Res., 88, 853-861, 1983b.

Rème, H., Aoustin, C., Bosqued, J. M., et al.: First multispacecraft ion measurements in and near the Earth's magnetosphere with the identical Cluster ion spectrometry (CIS) experiment, Ann. Geophysicae, 19, 1303-1354, 2001.

Russell, C. T. and Elphic, C.: ISEE observations of flux transfer events at the dayside magnetopause, Geophys. Res. Lett., 6, 3336, 1979.

Seon, J., Frank, L. A., Lazarus, A. J., and Lepping, R. P.: Surface waves on the tailward flanks of the Earth's magnetopause, J. Geophys. Res., 100, A7, 11 907-11 922, 1995.

Sibeck, D. G., Paschmann, G., Treumann, R. A., Fuselier, S. A., Lennartsson, W., Lockwood, M., Lundin, R., Ogilvie, K. W., Onsager, T. G., Phan, T.-D., Roth, M., Scholer, M., Sckopke, N., Stasiewicz, K., and Yamauchi, M.: Plasma transfer processes at the magnetopause, Space Sci. Rev., 88, 207-283, 1999.

Southwood, D. J.: The hydromagnetic stability of the magnetospheric boundary, Planet. Space Sci., 16, 587-605, 1968.

Southwood, D. J. and Hughes, W. J.: Theory of hydromagnetic waves in the magnetosphere, Space Sci. Rev., 35, 301-366, 1983.

Spreiter, J. R. and Stahara, S. S.: A new predictive model for determining solar wind-terrestrial planet interactions, J. Geophys. Res., 85, 6769-6777, 1980.

Treumann, R. A., Labelle, J., and Bauer, T. M.: Diffusion Processes: An Observation Perspective, in: Physics of the Magnetopause, edited by Song, P., Sonnerup, B. U., Ö., and Thomsen, M. F., AGU monograph, 331-341, 1995.

Treumann, R. A. and Baumjohann, W.: Advanced Space Plasma Physics, Imperial College Press, 1997. 